\title{
Foraging constraints reverse the scaling of activity time in carnivores
}

\author{
Matteo Rizzuto ${ }^{1, *}$, Chris Carbone ${ }^{2}$, Samraat Pawar ${ }^{1, \dagger}$ \\ ${ }^{1}$ Department of Life Sciences, Imperial College London, Silwood Park Campus, Ascot, UK \\ ${ }^{2}$ Institute of Zoology, Zoological Society of London, Regent's Park, London, UK
}

*Corresponding author. Current Address: Department of Biology, Memorial University of Newfoundland,230 Elizabeth Ave, St. John's, Canada; E-mail: mrizzuto@mun.ca

${ }^{\dagger}$ Corresponding author.E-mail: s.pawar@imperial.ac.uk

Running title: Scaling of daily activity time in carnivores

Keywords: Body size, Activity, Scaling, Metabolism, Movement, Animals, Carnivores, Biomechanics, Predator-prey, Foraging

Type of article: Article

Number of words in abstract: 163

Number of words in main text, excluding Methods: 560 (Introduction) +1478 (Results)

+1888 (Discussion) $=3926$

Number of words in Methods: 1118

Number of references: 43 (including those in Methods)

Number of tables: 1

Number of figures: 3

Name and contact of the person to whom correspondence should be sent: Samraat Pawar, Department of Life Sciences, Imperial College, London, Silwood Park Campus, Ascot, UK; Tel: +44(0)7863059770; email: s.pawar@imperial.ac.uk

Statement of authorship: All three authors designed the study. SP developed the mathematical model, and MR performed the data compilation and analyses. MR wrote the first draft of the manuscript; all three authors substantially revised the manuscript.

Acknowledgements: We would like to thank three anonymous reviewers for their detailed and helpful comments that greatly improved this paper. We would also like to thank Vito Muggeo and Dimitrios-Georgios Kontopoulos for their advice on the phylogenetically independent contrast and phylogenetic piecewise regression analyses. SP was supported by Grant NE/M004740/1 awarded by the National Environmental Research Council (NERC), UK, and the Grand Challenges in Ecosystems and the Environment (GCEE) Initiative at at Imperial College London. 


\begin{abstract}
The proportion of time an animal spends actively foraging in a day determines its longterm fitness. Here, we derive a general mathematical model for the scaling of this activity time with body size in consumers. We show that this scaling can change from positive (increasing with size) to negative (decreasing with size) if detectability and availability of preferred prey sizes is a limiting factor. These predictions are supported by a global dataset on 73 terrestrial carnivore species from 8 families spanning $>3$ orders of magnitude in size. Carnivores weighing $\sim 5 \mathrm{~kg}$ experience high foraging costs because their diets include significant proportions of relatively small (invertebrate) prey, and therefore show an increase in activity time with size. This shifts to a negative scaling in larger carnivores as they shift to foraging on less-costly vertebrate prey. Our model can be generalized to other classes of terrestrial and aquatic consumers, and offers a general framework for mechanistically linking body size to population fitness and vulnerability in consumers.
\end{abstract}




\section{Introduction}

The activity budget of an animal, that is, the time that it allocates to different behaviors on a daily basis, strongly affects its fitness by determining interaction rates with its resources, predators and competitors. The activity budget can also inform conservation efforts by helping predict the spatial and temporal distribution of resources necessary for an animal population to remain viable under habitat loss or climate change ${ }^{1,2,3}$. For example, accurate models of longterm activity and geographical ranges of mammalian carnivores are necessary for determining the appropriate size of protected areas of endangered mammals ${ }^{1,4}$.

However, although empirical patterns of activity budgets in the field are now widely recorded due to improved tracking technologies, there is currently no theoretical framework for predicting them, or generalizing our knowledge of these patterns across species, habitats, or foraging strategies. In particular, although animals can vary widely in how they manage their time, there is great potential for developing predictive models for activity budgets by including general constraints due to biomechanical (e.g., locomotion and searching) and energetic (e.g., basal or resting metabolic rate) limitations ${ }^{5,6,7}$.

Activity time on a daily basis should be strongly related to the minimum energetic requirements of an animal - more the energy requirement per unit time, more the time spent actively foraging for resources. An animal's energetic requirements can be estimated from its metabolic rate, which scales positively with body mass $(m)$. In the case of vertebrates, this scaling ranges between $m^{0.65}$ (for field metabolic rate) and $m^{0.9}$ (for active metabolic rate) ${ }^{8,9,10,11}$. Therefore, metabolic rate per unit body mass (mass-specific metabolic rate) in vertebrates scales negatively with body size with an exponent ranging between -0.35 and -0.1 . That is, individuals from small-sized species have higher maintenance costs per unit body mass and necessarily need to consume energy at a faster rate than those from larger ones. In other words, all else being equal, smaller vertebrates need to forage for longer periods than larger ones. This leads to a simple prediction: the amount of time a vertebrate spends active in its daily cycle also scales with body mass within the range $m^{-0.35}-m^{-0.1}$.

However, this prediction of a negative scaling of activity time with body size hinges on the key assumption that energy intake rate scales identically to the rate of energy use (metabolic rate). In reality however, intake rates in the field are typically limited by resource availability (i.e., prey abundance), and ability of the consumer to search, detect, attack and handle prey. This can result in deviations of the scaling of intake rate from that of metabolic rate rep $^{12,13,15}$. Because of these constraints on field intake rates, animals of different sizes need to optimize their activity budget by choosing the right resource sizes (e.g., many small vs. few large prey) and foraging strategy (e.g., active-capture vs. sit-and-wait) to meet their energetic needs ${ }^{16,13,17}$. Thus these limitations on foraging, and therefore intake rates, may ultimately lead to deviations from the expectation of a universal decrease in activity time with body size.

Here we derive a general mathematical model for the size-scaling of the activity budget of consumers under field conditions, incorporating key metabolic and biomechanical constraints on foraging costs. We then develop a specification of the model appropriate for terrestrial mammalian carnivores, and test its predictions by compiling a global dataset of high-resolution activity budgets. In the Discussion we show how our model can be specified or extended to other classes of consumers.

\section{Results}

Our model links consumer and resource body size to the minimum proportion of time $\left(T_{p}\right)$ that an individual consumer must spend foraging on a daily basis to maintain its energy balance (see 
Methods, and Supplementary Information for detailed derivation):

$$
T_{p}=\frac{B_{0} m^{\beta}}{B_{0} m^{\beta}-A_{0} m^{\alpha}+I_{0} m^{\iota}}
$$

Here, $m$ is an individual consumer's adult average body mass (its size), $I_{0}, B_{0}$, and $A_{0}$ are the standard (i.e., for a $1 \mathrm{~kg}$ consumer) intake, resting metabolic, and active metabolic rates respectively, while the scaling exponents $\iota, \beta$ and $\alpha$ respectively quantify the size-dependence of these three fundamental rates. This model makes a key prediction (see Methods and Supplementary Information for derivation): if $l$ is the exponent of the scaling of energy loss (either while resting or actively foraging), if

$$
\iota<l,
$$

the scaling relationship of $T_{p}$ with body size changes from negative ( $T_{p}$ decreasing with size) to positive ( $T_{p}$ increasing with size). The critical value $l$ for the intake rate scaling exponent (at which the scaling relationship reverses) is expected to lie between approximately 0.70 if resting (through the exponent $\beta$ ) dominates energy expenditure, and 0.80 if active foraging (through the exponent $\alpha$ ) dominates. This result about the reversal of activity time scaling remains robust to considerable variation in scaling exponents due to uncertainty in their estimation as well as biological variation such as differences in the scaling of basal, field, and maximum metabolic rates (Supplementary Information).

As such, equation (1) and the prediction of a critical value of intake rate scaling (equation (2)) provides an simple, intuitive model for determining the necessary intake rate scaling to maintain a negative scaling of activity time with increasing body size. Furthermore, for a given scaling of active and resting metabolic rates equation (1) can be used to estimate the the body mass threshold below which the scaling exponent of intake rate must increase to maintain activity time below a biologically feasible limit (for example, assuming that the daily activity time proportion cannot exceed, say, 0.5).

Next, to obtain a mechanistic basis for the constraints on intake rate scaling and determine where the body mass threshold for a qualitative change in the daily activity proportion may lie under field conditions, we show that $\iota$ can be broken down into the contributions of different constraints on foraging, and therefore intake rate:

$$
\iota=p_{v}+p_{x}+\left(p_{k}+1\right) p_{d}
$$

Here, $p_{v}$ is the size-scaling exponent of body velocity, $p_{x}$ the exponent of prey abundance, $p_{k}$ is the exponent of resource size relative to consumer size, i.e., the resource-consumer (e.g., preypredator) size ratio, and $p_{d}$ is the exponent of detection distance. Of these, $p_{x}, p_{k}$, and $p_{d}$ are most important because body velocity (through its scaling exponent $p_{v}$ ) contributes to both energy gain and loss while actively foraging. These are ubiquitous constraints imposed by field conditions on the intake rate and therefore activity time: the prey-predator size-ratio scaling determines the effect of availability of or preference for resources of different sizes relative to predator, reaction distance scaling determines the effect of detectability of these resources, and abundance scaling determines the effect of prey rarity which translates into higher foraging costs by decreasing the number of resource encounters per unit time spent foraging. These foraging constraints can be interpreted partly as inherent, biomechanical constraints of consumers, and partly as properties of the local ecological conditions, which may constrain availability of prey of different sizes.

An example of the effect of each of these three foraging constraints on the predicted scaling of intake rate and therefore, activity time, is shown in Fig. 1. To generate these predictions, we use size-scaling relationships for energy loss rates $(B$ and $A)$ and components of intake rate $(I)$ appropriate for terrestrial mammalian carnivores, as these have been extensively studied ${ }^{16,19}$. This yields the prediction that terrestrial carnivores are expected to have a scaling of activity 

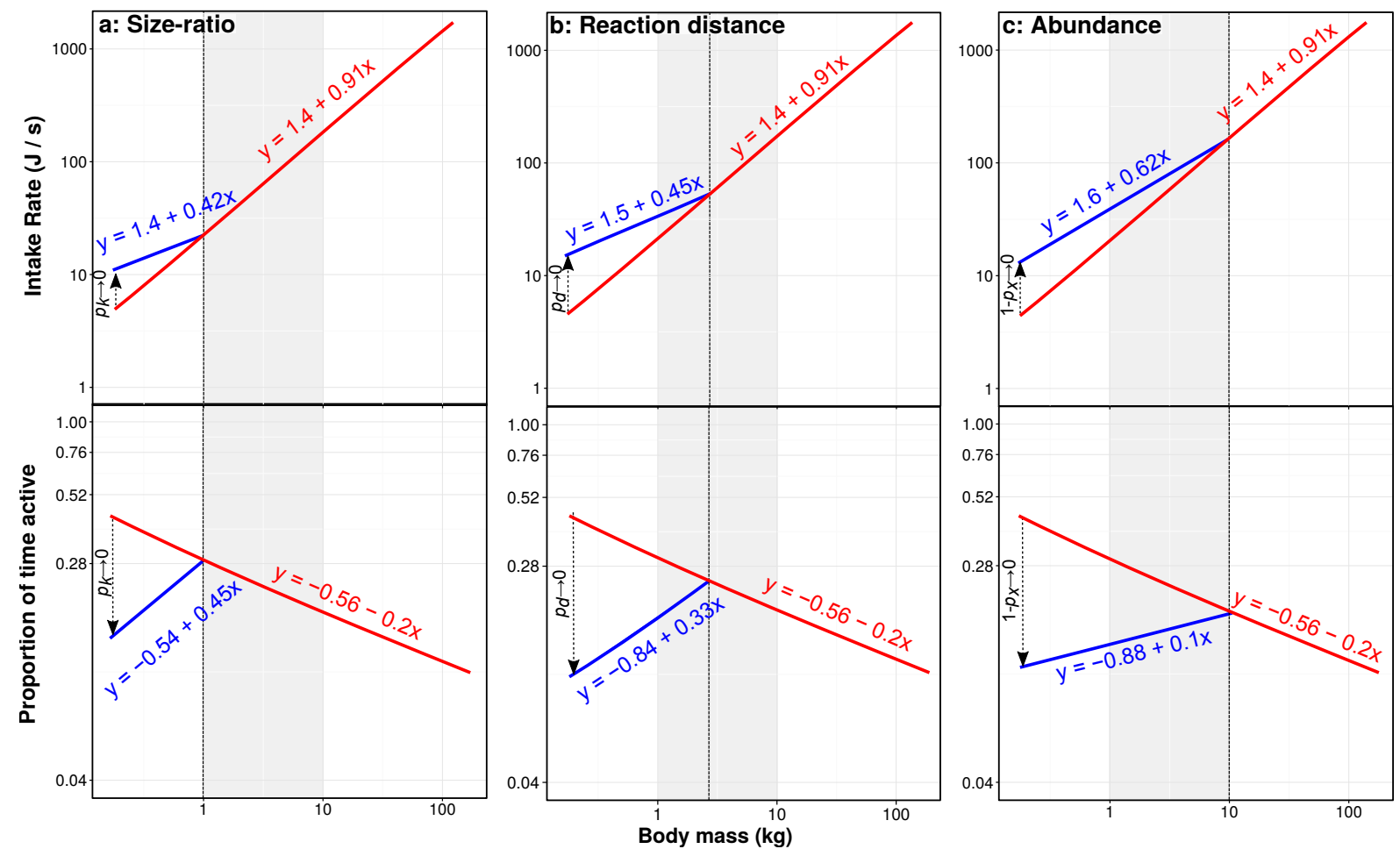

Figure 1: The size-scaling model of activity time in terrestrial carnivores. The three panels show effect on scaling of the activity time (lower figures), due to a weakening of intake rate scaling $\iota$ (upper figures) through three constraints of field conditions: a. Scaling of preypredator size-ratio $p_{k}$, which captures the constraint of availability of suitable (relative) prey size with increasing predator size; b. Scaling of reaction distance $p_{d}$, which captures the constraint on ability of predators to detect prey of a certain size relative to themselves; c. Scaling of prey biomass abundance $p_{x}$, which captures the constraint of availability of sufficient prey individuals with increasing predator size. In all plots, blue lines represent the deviation of scaling of intake rate or activity budget from the "normal" scaling (red lines) — when none of these constraints exist. Note that all the intersection points of pairs of scaling (red and blue) lines lie between 1-10 kg predator weight range (highlighted).

time with an exponent of $\approx-0.2$ (Fig. 1 ), which changes to a positive scaling exponent if foraging is subject to one or all of three biomechanical constraints: either size, reaction distance, or abundance of preferred prey do not keep up with increasing carnivore size. The precise value of the positive scaling exponent depends upon which of these constraints applies and to what degree. Underlying this is the scaling of intake rate, predicted to be $\approx 0.9$, which changes to an exponent between $0.4-0.65$ depending on the type and strength of the same biomechanical constraints. The intersection between the two scaling relationships occurs between $\approx 1-10 \mathrm{~kg}$ carnivore size.

Thus our model provides a nuanced, mechanistic explanation for the costs of foraging in small carnivores, and links these costs to the scaling of activity time in small vs. large terrestrial carnivores. Specification of the general model (equation (1)) for other types of consumers will likely yield different scaling predictions for intake rate (equation (3)) and activity time scaling (equation (2)).

Next, to test our theoretical predictions, we compiled independent datasets on intake rates and activity budgets on 73 species of terrestrial carnivores spanning most of the extant size of the Order (Supplementary Information). From these data, we first calculated the proportion of time spent active $\left(T_{p}\right)$ in a day across 38 carnivore species. We restricted the activity budget 


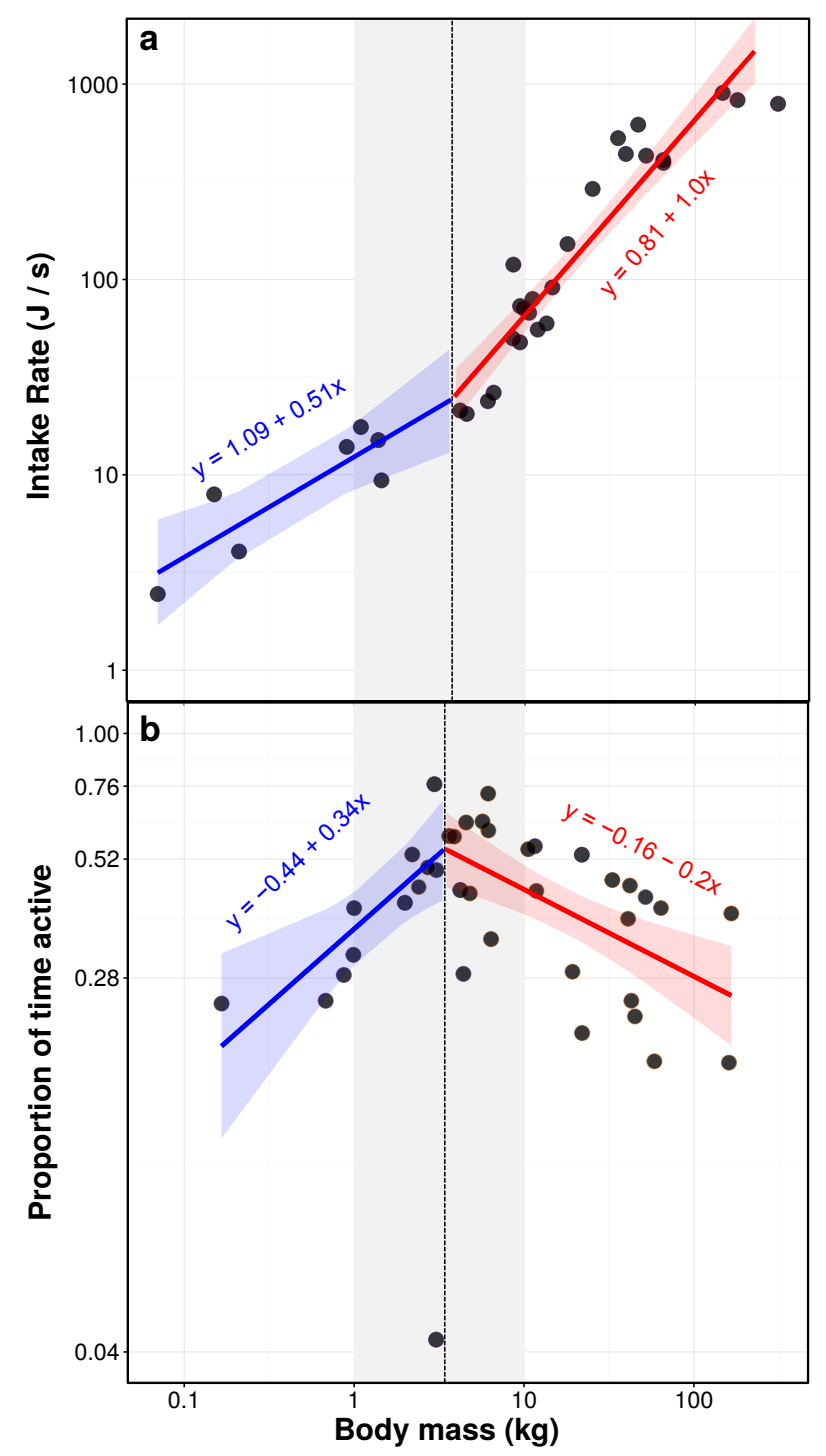

Figure 2: Observed size-scaling of Energy Intake Rate and Activity Proportions among Carnivores. In both panels, the blue and red lines represent the two segments of the breakpoint regression (with $95 \%$ prediction bounds), the vertical line is their intersection (the breakpoint), and the vertical shaded area the 1-10 $\mathrm{kg}$ interval predicted by the theory (Fig. 1). a: The scaling of energy intake rate $(n=32$ species). Breakpoint is at $3.74 \mathrm{~kg}$, and the two scaling exponents (i.e., the slopes) are significantly different (Davies' test, $p=0.014$ ). b: Activity budget scaling ( $n=38$ species). Breakpoint is at $3.42 \mathrm{~kg}$, and the two scaling exponents are significantly different (Davies' test, $p=0.0006)$. The outlier represents activity data of one Egyptian mongoose (Herpestes ichneumon) collected over the course of 1 month in Spain (Supplementary Information). Also see Fig. S5 \& S10 for species- and family-level contributions to these results. 
data compilation to biotelemetry and GPS tracking studies because other methods (e.g., cameratrapping, direct observation) are likely to under sample the proportion of time active in smaller animals due do their poorer temporal and spatial resolution. We find that there is an overall tent-shaped relationship between $T_{p}$ and carnivore body size across the entire size range (Fig. 2). Larger carnivore species generally become less active ( $T_{p}$ decreases) as body size increases, while smaller carnivores become more active ( $T_{p}$ increases) as body size increases.

Table 1: Parameter values for the piecewise regression model fitted to the Activity Time, Intake Rate and Size Ratio data vs. Body Mass data. All variables were $\log _{10^{-}}$ transformed. The $\triangle \mathrm{AICc}$ is the difference in the small-sample size Akaike Information Criterion value for the piecewise ordinary least squares (OLS) vs. a single-line OLS model fitted to the data. The intercept and slope ( $\pm 95 \%$ Confidence Intervals $)$ pairs are for the fitted OLS models below and above the breakpoint (cf. Fig. $2 \& 3$ ). Further details on the model fitting and selection results are in the Supplementary Information.

\begin{tabular}{llll}
\hline & Activity proportion & Intake Rate & Size Ratio \\
\hline$\Delta \mathrm{AICc}$ & -13.9 & -6.54 & -10.6 \\
$r^{2}$ & 0.43 & 0.94 & 0.55 \\
Breakpoint & $0.53 \pm 0.14$ & $0.57 \pm 0.34$ & $0.68 \pm 0.20$ \\
Intercepts & $-0.44,-0.16$ & $1.09,0.81$ & $-1.24,-2.74$ \\
Slopes & $0.34 \pm 0.11,-0.20 \pm 0.05$ & $0.51 \pm 0.15,1.00 \pm 0.08$ & $0.21 \pm 0.44,2.41 \pm 0.36$ \\
\hline
\end{tabular}

This overall pattern is best explained by a piecewise regression (Table 1, Supplementary Table S2). The breakpoint — the body weight where the scaling relationship reverses - is estimated to be at $3.42 \mathrm{~kg}$ (95\% confidence intervals (CIs): $1.79-6.52)$, within the $1-10 \mathrm{~kg}$ range predicted by our model (cf. Fig. $1 \& 2$ ). This breakpoint is around the average weight of a Gray Fox (Urocyon cinereoargenteus). The slopes of the piecewise model's left and right segments were also significantly different $(0.34 \pm 0.11$, and $-0.2 \pm 0.18$ respectively, $p<0.0001)$ (Table 1). Thus, the empirically-observed exponent for the activity time for large carnivores is statistically indistinguishable from the value predicted by our model. The value of the exponent for small carnivores $(0.34 \pm 0.11)$ is also within the range predicted by the model (Fig. 1).

Next, we used the dataset on intake rates (32 species) to test the mechanistic link between the scaling of intake rate and activity time predicted by our theory (Fig. 1; equations (2) \& (3)). Figure 2 shows strong support for our prediction: a change in activity time scaling is coupled with a significant weakening of the intake rate scaling in smaller carnivores (Fig. 1). The qualitative change in the intake rate scaling takes place at $\sim 3.74 \mathrm{~kg}(95 \%$ CIs: $0.86-8.34)$, statistically indistinguishable from the breakpoint for the scaling of the activity budget (Fig. 2). The slopes of the piecewise model's two segments, to the left and to the right of the breakpoint, differed significantly $(0.51 \pm 0.29$ and $1 \pm 0.15$ respectively, $p=0.013)$ (Table 1$)$. Furthermore, in the upper panel of Fig. 2, the slope to the left of the breakpoint is below the 0.7-0.8 value predicted by our theory (the exponent $\alpha$; equation (2)). Thus overall, we find strong support for the predicted mechanistic link between intake rate and activity time.

We did not detect a significant phylogenetic signal in the activity budget or intake rate datasets (Supplementary Information). The results also remain qualitatively unchanged after fitting a linear mixed effect model to the data with study and species identity as random effects (Supplementary Information), nor does accounting for seasonal resource availability or reanalyzing the data for only the three most data-rich Families (Supplementary Information).

Thus our results indicate strong constraints on intake (foraging) rates, and therefore on activity times in small terrestrial carnivores (below a body size of $\sim 5 \mathrm{~kg}$ ). These constraints arise in small carnivores because some or all of the following: prey biomass abundance increases too weakly with increasing predator body mass, prey body sizes increase too weakly (that is, 
larger species among small carnivores feed on prey that are sub-optimally small), or reaction distance does not increase or increases too weakly (Fig. 1).

To gain further insights into which of these constraints might dominate the observed patterns in activity budget scaling, we compiled a third independent dataset on prey-predator body sizes relationships for terrestrial carnivores (Supplementary Information). We tested whether smaller carnivores fed on qualitatively different relative prey sizes than larger carnivores. The results (Fig. 3) show that there is indeed a significant shift in the relative size of preferred prey between small and large carnivores - prey size scales strongly and positively with body size in large carnivores, but not in small carnivores. A breakpoint regression indicates that the shift lies within the $1-10 \mathrm{~kg}$ size range, at about $4.8 \mathrm{~kg}$ (95\% CIs: $2.34-7.25)$ a value that is somewhat larger but statistically indistinguishable from the breakpoints for the activity budget and intake rate scaling relationships (Fig. 2). Thus, the fact that smaller terrestrial carnivores tend to feed on prey items of relatively constant size that are much smaller then themselves (Fig. 3) at least partly explains the reversal of scaling of the activity budget.

\section{Discussion}

We have developed a mechanistic model to predict the body size-scaling of the time consumers need to be active (i.e., foraging) for maintaining energetic balance. By specifying this model for terrestrial carnivores and by analyzing a global empirical dataset, we find that somewhat counter-intuitively, small and large terrestrial carnivores have an opposite scaling of the proportion of time spent active $\left(T_{p}\right)$ in a day. Specifically, small carnivores below a $1-10 \mathrm{~kg}$ size range show an increase in daily activity time with body size, with only larger carnivores showing the decrease in activity time with size expected from their lower mass-specific energy needs ${ }^{5}$. This reversal of scaling occurs because small carnivores face additional constraints while foraging, which limits their intake rate and negates the advantage of decreasing mass-specific metabolic rates with increasing size. These results also provide an explicit, (bio)mechanistic, and empirically-validated theoretical model for the cost of small-prey eating hypothesized by Carbone et $\mathrm{al}^{19,16}$, who predicted a similar upper threshold size $(14.5 \mathrm{~kg})$ for small prey eating.

The three constraints - prey-predator size ratio, reaction distance, and resource abundance - are general in that all of them are likely to be experienced by predators under field conditions. That is, unlike in larger carnivores, where bigger species feed on proportionally bigger prey, in small carnivores preferred prey size changes little as body size increases, as can be seen in Fig. 3. Thus, in small carnivores, though foraging on small prey is initially relatively easy due to low hunting costs, as predator size increases, prey become increasingly difficult to detect, attack and handle.

Size-ratio and reaction distance are tightly interlinked, because smaller size-ratios (prey much smaller than predator) also decrease reaction distance ${ }^{12,13}$. Current models and data suggest that reaction distances scale positively with body size when considering visual constraints, so that larger species have bigger reaction distances ${ }^{23,12,13}$. However, field conditions impose multiple constraints on how far an animal can see, including vantage point, line of sight, prey conspicuousness and maneuverability, all of which likely raise additional challenges for small predators hunting for much smaller prey. Indeed, it has been suggested that natural habitats show fractal (i.e., self-similar) visual structure ${ }^{24}$ which implies that, compared to large-prey eaters, small-prey eaters hunt for better-hidden prey in effectively more complex landscapes.

Thus, although data are currently lacking on the scaling of reaction distance in terrestrial carnivores, the weak scaling of both intake rates and prey sizes seen in small carnivores indicates that species up to about $10 \mathrm{~kg}$ face somewhat insurmountable challenges in the way of feeding on optimally-sized prey. This is likely compounded by the increasing costs of rapid maneuvering necessary for small-prey hunting ${ }^{21,22}$, and greater prevalence of nocturnal foraging (which presumably also limits prey detectability) in small carnivores. Carnivore species above the 1- 


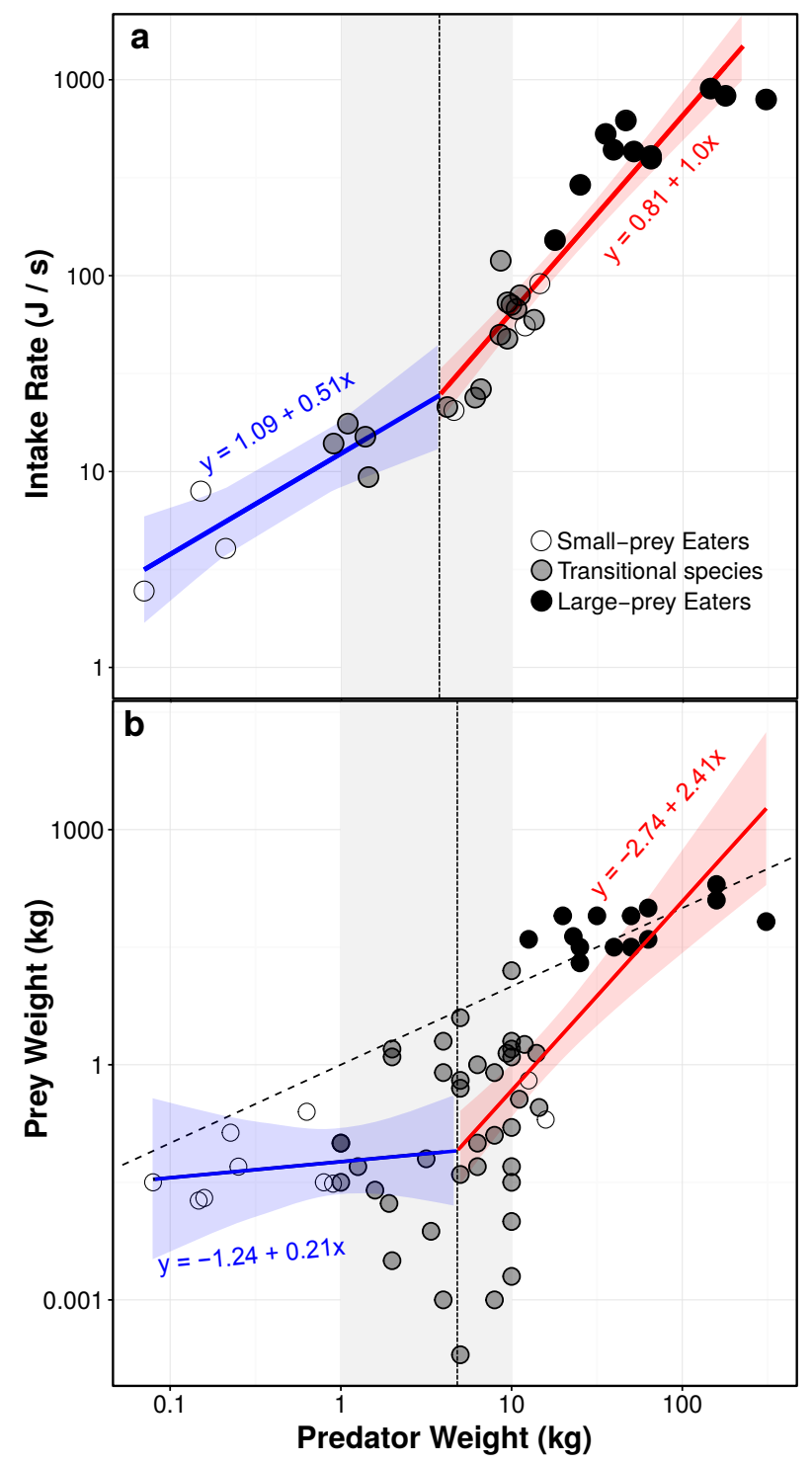

Figure 3: Scaling of intake rate and prey vs. predator size (size-ratio) among carnivores. In both panels, the blue and red lines represent the two segments of the breakpoint regression (with $95 \%$ prediction bounds), the vertical line is their intersection (the breakpoint), and the vertical shaded area the 1-10kg interval predicted by the theory (Fig. 1). a: Same as Fig. 2a, but with the data classified by relative prey-size based feeding strategies instead of taxonomy. b: scaling of prey with predator size ( $n=63$ species). The breakpoint estimated here is $4.8 \mathrm{~kg}$ : above this, size of preferred prey scales positively with predator size, whereas below this, prey size remains roughly constant even though consumer body mass increases. The two slopes are significantly different (Davies' test, $p=0.0025$ ). The diagonal dashed line represents the predator-prey sizes where the size-ratio equals 1; values below it indicate prey are smaller than the predator. The classes of prey-size based feeding strategies shown were defined on the basis of the range of prey types taken, as explained in Supplementary Information Section 2.4. Note that in both plots, the transitional species lie largely within the 1-10kg (shaded) range. 
10kg size range escape these constraints by feeding on relatively larger prey ${ }^{19,20}$ (Fig. 3), which results in a switch to a steeper scaling of intake rate and correspondingly, a negative scaling of activity time (Fig. 2).

Handling time, which includes the time spent in pursuing and capturing prey ${ }^{15}$ following detection and reaction, would be subject to similar constraints. Handling rates are unimodal with respect to prey-predator size ratios, that is, they decline at both extremes of prey-predator body size ratios ${ }^{16,25,20}$. Therefore, the main advantage of feeding on small prey - a relatively short handling time ${ }^{16,20}$ - is increasingly negated for larger small-prey eaters as their prey become sub-optimally smaller relative to themselves. This would compound with the constraints of reaction distance. Future work should aim to directly test this hypothesized weaker scaling of reaction distance among small carnivores, as well as the variation in handling time scaling across different size-classes of carnivores.

A weak scaling of prey abundance can accentuate the constraints faced by small carnivores (Fig. 1). Resource biomass abundance, which scales positively with resource body mass and therefore also predator body mass, (the exponent $1-p_{x}$ in equation (7), Supplementary Information equation S13), can partly offset the higher cost of foraging for small prey in small carnivores. If the numerical abundance of the prey (exponent $p_{x}$ ) of small-prey eaters itself scaled more strongly, such that larger prey items were rarer, small-prey eating would become more costly with increasing predator size, leading to an even stronger positive scaling of the proportion of time active with size. Whether, in general, this is true within the size range of prey species relevant to small carnivores is currently unknown. Therefore, future work should also aim to quantify the scaling of abundance of target prey for different size-classes of carnivores.

The upshot of these constraints on foraging is that in order to maintain energetic balance, small-prey eaters have to start preferentially taking larger prey beyond a certain body size range ${ }^{16}$, or evolve morphological and behavioral specializations to feed on small prey (e.g., the Aardwolf; Proteles cristata $\left.{ }^{26}\right)$. In this regard, it is notable that we do find an abrupt diversification of prey sizes taken (including the Aardwolf example) within the $1-10 \mathrm{~kg}$ size range (the shaded area in Fig. 3, consisting mostly of "transitional" species). This may explain why small carnivores (Viverrids, as well as many Canids and Felids) in the 1-10 kg body weight range increase the diversity of size range of their prey - becoming more generalized to offset the increased costs of foraging. A signature of these forging constraints on small carnivore foraging may also be seen in long-term home range size and usage. Understanding animal home range sizes and usage is important for design of protected areas ${ }^{1,4}$, and is a promising avenue for future work based on the findings of this study. Also, because the same biomechanical constraints highlighted here for small-prey eaters could apply to large-prey eating terrestrial carnivores if sufficiently large prey are unavailable (or go extinct), our model may also provide a mechanistic explanation for body size limits to large carnivores, and why gigantic forms in many extant carnivore families have appeared and become extinct time and again in the paleontological record ${ }^{16,27}$.

Our results thus shed light on the behavioral adaptations involved in offsetting the higher energetic requirements of increasing size, and reveal ecological challenges faced by small carnivores. Small carnivores may be particularly susceptible to habitat degradation if this leads to an increase in foraging activity and therefore adds to an already sub-optimal activity budget. As human-induced environmental changes become ever more common and severe, these species may be among the first and more seriously affected - calling for further studies to inform adequate conservation policies. From this perspective, the modelling framework we propose here can be used to develop a better understanding or a priori predictions for daily activity times within individual species across their geographical range. In particular, by appropriately parameterizing the resource (prey) abundance scaling constant $x_{0}$, the model can be used to predict how different populations of the same species respond to spatial or temporal variability in resource availability and quality over it's geographical range. For example, the percentage 
increase in daily activity time due to a decline in resource abundance over time or space for a species can be predicted using the model, and then tested using field data. Similarly, by appropriately parameterizing the size-ratio scaling constant $k_{0}$ and exponent $p_{k}$, the model can be used to predict the effect of variation in availability of appropriate or preferred prey on activity budgets of a species across its range. This would provide key insights into threats to species' energy budgets and therefore, ultimately population sizes and sizes of protected areas necessary for maintaining a "healthy" activity budget in a target species.

Also, to develop such accurate, species-specific predictions, more realistic parameterizations of the cost of locomotion will be be needed. For example, the equation for scaling of the cost of locomotion we use ${ }^{18}$ (equation (6)) likely underestimates the actual metabolic costs experienced by carnivores in the field. Therefore, we would expect a higher intercept of the scaling of activity time than predicted here (Fig. 1). The increasingly cheaper techniques available for field measures of carnivore energetics hold great promise for more accurate predictions for specific species or groups of species (e.g., mustelids vs. canids and felids; see SI section 2.5.6) using this modelling framework.

Another source of variation in daily activity time is likely to be the seasonal changes in energy requirements for breeding (e.g., searching for mates and defending territories) and overwintering (e.g., storing fat for hibernation) in many species. Therefore, though our result about the dual scaling of activity time remains qualitatively robust across resource-rich and resource-poor seasons (SI section 2.5.6 \& Fig. S9), elaborating our model to include seasonal energy loss terms will allow a more accurate predictions of activity time. This will have to be coupled with tracking datasets at sufficient resolution to allow a proper investigation of the effects of seasonal bursts or declines in daily energy expenditure on activity patterns. For this, the general bias towards relatively larger carnivore species evident in the published literature on activity patterns (and therefore also in our compiled data; see Fig. 2) needs to be addressed first. The results of this study emphasizes the need for some correction of focus of tracking studies from bigger, charismatic carnivores to smaller, more elusive species.

From a more theoretical perspective, our model framework could be adapted and extended to explore the role of biomechanical constraints in the field on activity budgets for a wide range of organisms, opening up research avenues for understanding links between behavioral and population processes. Specifically, using appropriate scaling models for reaction distance and resource abundance, the model can be extended to herbivores, invertebrate predators, or consumers that forage in three spatial dimensions $\left(3 D\right.$; such as in pelagic environments ${ }^{13,14,17}$. Extending the model to ectothermic consumers (e.g., all invertebrate predators) would require appropriate models for temperature dependence of metabolic rates and body velocity ${ }^{28,15}$. For example, to generate predictions for aquatic predators, the equations and parameter values for the energy costs of inertial aquatic locomotion (equation (6)) and basal metabolic rate (equation (5)) for aquatic organisms could be used, coupled with a change from a $2 D$ to $3 D$ intake rate model ${ }^{13}$. Although herbivores may be less constrained by reaction distance, they may still be subject to size-ratio or resource abundance scaling constraints on intake rate ${ }^{29}$. As recent studies on other classes of mammals both substantiate ${ }^{30}$ and contradict $^{31}$ the dual scaling relationship of activity budget with body size found in the present work, our results prompt further, in-depth investigation of the effects these constraints have on non-carnivorous mammals.

In conclusion, the proportion of time that animals need to dedicate to foraging depends upon the biomechanical constraints they face in the field. We find strong evidence that small terrestrial carnivores face such constraints, likely arising from a combination of sub-optimal prey-predator size ratios, weak scaling of reaction (effective detection) distance and possibly, prey abundance. These constraints change the energetic advantage of increasing body size to a disadvantage, and the scaling of proportion of activity time reverses in small vs. large carnivores. By quantifying the mechanistic links between field conditions and activity budgets, our model 
offers insights into the constraints on animal fitness in the field, and what foraging strategies may be preferred in different biotic and abiotic contexts. Further work along these lines would provide field scientists, conservationists, and theoreticians with a powerful tool with which to explore how species adjust to both environmental and physiological changes, expanding on our knowledge of the ecology, evolution, and conservation of as of yet poorly understood consumer species and consumer-resource interactions.

\section{Methods}

Model development. We start by deriving a model for the minimum proportion of time $\left(T_{p}\right)$ that an individual consumer must spend foraging on a daily basis to maintain its energy balance (see Supplementary Information for derivation):

$$
T_{p}=\frac{B}{I+B-A}
$$

where $I$ as the individual's energy intake rate, $B$ its energy loss rate when resting and $A$ its energy loss rate when active. All rates are in $J \cdot s^{-1}$. We then impose biomechanical and metabolic constraints on the three components of equation (4) using metabolic scaling theory ${ }^{5,8,13,15}$ appropriate for terrestrial carnivores (Supplementary Information for further details). Specifically, for energy loss rate while resting, we use the scaling of basal metabolic rate,

$$
B=B_{0} m^{\beta}
$$

Where $m$ is the body mass (in $\mathrm{kg}$ ) of the predator. For energy loss during movement, we use Taylor et al.'s model ${ }^{18}$ (see Supplementary Information for more details):

$$
A=A_{0,1} v_{0} m^{a_{1}+p_{v}}+A_{0,2} m^{a_{2}}
$$

Where $v_{0}, A_{0,1}$, and $A_{0,2}$ are constants (Supplementary Table S1). The first term of the sum $\left(A_{0,1} v_{0} m^{a_{1}+p_{v}}\right)$ quantifies the increase in energy consumption during movement as a function of body size (exponent $a_{1}$ ) and body velocity (exponent $p_{v}$ ) (incremental cost), while the second term $\left(A_{0,2} m^{a_{2}}\right)$ quantifies the energy needed to initiate the movement (zero speed cost). For energy intake rate we use the consumption-rate model derived for individuals foraging in $2 D$ (two euclidean dimensions) $)^{13,15}$,

$$
I=I_{0} m^{\iota}
$$

where

$$
I_{0}=2 v_{0} d_{0} x_{0} k_{0}^{1-p_{x}+p_{d}}
$$

and

$$
\iota=p_{v}+p_{x}+\left(p_{k}+1\right) p_{d}
$$

Here, $p_{v}, p_{d}$ and $1-p_{x}$ are the scaling exponents of the predator's velocity (same as in equation (6)), reaction distance and prey biomass abundance respectively. Reaction distance is the minimum distance at which the consumer can detect the prey and react to it ${ }^{13}$. The exponent $p_{k}$ is for the scaling of prey-predator body size-ratio $\left(\frac{m_{R}}{m}\right) . I_{0}$ is the product of the scaling constants of velocity $\left(v_{0}\right)$, reaction distance $\left(d_{0}\right)$, size-ratio $\left(k_{0}^{p_{d}}\right)$, and prey biomass abundance $\left(x_{0}\right)$. Substituting equations eqs. (5) to (7) into equation (4) followed by some simplifications and approximations (detailed in Supplementary Information) gives the biomechanically-constrained activity budget model (equation (1)). Analysis of this model to determine the inflection point, which satisfies the condition $\frac{d \log \left(T_{p}\right)}{d m}=0$, yields the result shown in equation (2).

Model parameterization. We provide a detailed account of the model parameterization in the Supplementary Information, along with values of all scaling parameters. In short, we used 
published scaling relationships for all variables in equation (1). We parameterized equation (5) by reanalyzing carnivores' data from Kolokotrones et al. ${ }^{8}$, after dropping aquatic and omnivore species. For equation (6), we used the values in the original paper by Taylor et al. ${ }^{18}$ re-expressed in $\mathrm{J} \cdot \mathrm{s}^{-1}$ from the original $\mathrm{J} \cdot(\mathrm{kg} \cdot \mathrm{s})^{-1}$. The scaling equation for intake rate $I$ involves three different relationships (equation (7)): we used the reaction distance equation from ${ }^{13}$, the sizeratio scaling relationship from ${ }^{5}$ and the prey biomass abundance models of ${ }^{16,13}$. For the velocity term $v$, which appears in both the scaling of $A$ and $I$, we used the relationship by ${ }^{43}$ as cited in $^{5}$. We used an energy content value of $1 \mathrm{~kg}$ wet mass $=7 \cdot 10^{6} \mathrm{~J}$ in all conversions ${ }^{5}$.

Sensitivity analyses. We tested our model for sensitivity to both variation in its mathematical structure and in the values of the parameters used. Results from the structural sensitivity analysis are shown in Supplementary Fig. S1 \& S2, and indicate that our carnivore model specification is robust to simplification of its mathematical structure. We also used equation (1) to determine the contribution of each of the three constraints (prey abundance, prey size and prey reaction distance) alone to the size-scaling of intake rate and activity budget (Fig. 1). As can be seen in Fig. 1, each of these constraints can by itself result in a qualitative (negative to positive) shift in the scaling of activity budget, with the shift occurring within a size range of about 1-10 kg predator body weight. Finally, we sampled each of our seven scaling exponents 10,000 times independently from a Gaussian distribution to test for robustness to variation in the parameterization of all scaling relationships. Each time, we re-calculated $I$ and $T_{p}$. Supplementary Fig. S3 shows the results of this analysis, highlighting that our main results are qualitatively robust to uncertainty in the values of our scaling parameterizations.

Data collection. We collected data on the daily activity budget of terrestrial carnivore species from both published literature and existing databases. We focused on data collected via high-resolution radio-tracking techniques (VHF, GPS and Accelerometers) to build a consistent dataset that would allow for direct comparison between different species and because of substantial variability in the accuracy of different techniques (Supplementary Information). We used a set of keywords defined a priori and selected only studies reporting full 24 hours activity cycles based on 1 or more complete years of sampling. Using open-source software ${ }^{34}$, we digitized graphs and tables, and then converted all data collected to SI units of time (s). We did not include marine (e.g., Pinnipeds) and omnivore (e.g., Ursids) species in our dataset. We used an existing dataset for intake rate data ${ }^{16}$. Similarly, when not available in the original sources, we used an existing dataset for average body weight ${ }^{35}$. We obtained size-ratio data (i.e, mass of predators and of their preferred prey) from the published literature ${ }^{19,16,36}$. We classified diet of carnivores species based on the percentages of different food categories present in their diet, and then classified them as either "large-prey eaters" or "small-prey eaters" (Supplementary Information).

Data analyses. We conducted all analyses in R (v. 3.3.0 ${ }^{37}$ ) with significance levels set as $\alpha=0.05$. Our dataset showed substantial pseudo-replication: we accounted for this by taking the geometric mean of repeated measures, which allowed us to obtain a single average value of activity over 24 hours for every species in our dataset (Supplementary Information). A special case of pseudo-replication is represented by phylogenetic relatedness ${ }^{38}$. To account for this, we tested both our activity times and intake rate datasets for phylogenetic signal using a recently published tree for carnivores ${ }^{39}$. Using R package "geiger" ${ }^{40}$, we fitted 3 models to each dataset: a maximum likelihood model, a brownian motion model with $\lambda=0$ and brownian motion model with $\lambda=0$. We used an information theory approach to establish the better model and found no evidence of phylogenetic signal in either dataset (Supplementary Information). To quantify the relationship between the activity times and body weight in our dataset, we fitted 3 different regression models to the $\log _{10}$-transformed variables: an ordinary least squares (OLS), a second degree (quadratic) polynomial and a piecewise regression using $\mathrm{R}$ package "segmented" 41 (Supplementary Information). To test for differences in the slopes of the two segments of the piecewise regression, we used the Davies' test ${ }^{41}$. Analyses of the intake rate 
and size-ratio data, as well as those on the effects of seasonality, followed similar procedures (Supplementary Information). We repeated these analyses on the un-transformed data, using a linear mixed model with Study and Species as random effect fitted using R package "nlme" 42 .

Code and Data Availability. The computer code and data used in the present analyses are available from Figshare public repositories (identifiers 10.6084/m9.figshare.5466295 and 10.6084/m9.figshare.5464150 respectively).

Competing Interests Statement. The authors declare no competing interests.

\section{References}

[1] Woodroffe, R. and Ginsberg, J. Edge effects and the extinction of populations inside protected areas. Science 280(5372), 2126-2128, jun (1998).

[2] Jetz, W., Carbone, C., Fulford, J., and Brown, J. H. The scaling of animal space use. Science 306(5694), 266-8, oct (2004).

[3] McCain, C. M. and King, S. R. B. Body size and activity times mediate mammalian responses to climate change. Glob. Chang. Biol. 20(6), 1760-1769, jun (2014).

[4] Miller, C., Hebblewhite, M., Petrunenko, Y., Seryodkin, I., Goodrich, J., and Miquelle, D. Amur tiger (Panthera tigris altaica) energetic requirements: Implications for conserving wild tigers. Biol. Conserv. 170, 120-129, feb (2014).

[5] Peters, R. The ecological implications of body size. Cambridge University Press, Cambridge, 1st edition, (1983).

[6] Schmidt-Nielsen, K. Scaling, why is animal size so important? Cambridge University Press, Cambridge ; New York, (1984).

[7] Gorman, M., Mills, M., Raath, J., and Speakman, J. High hunting costs make African wild dogs vulnerable to kleptoparasitism by hyaenas. Nature 852(1992), 1992-1994 (1998).

[8] Kolokotrones, T., Savage, V., Deeds, E. J., and Fontana, W. Curvature in metabolic scaling. Nature 464(7289), 753-6, apr (2010).

[9] Speakman, J. R. and Król, E. Maximal heat dissipation capacity and hyperthermia risk: Neglected key factors in the ecology of endotherms, , apr (2010).

[10] Hudson, L. N., Isaac, N. J. B., and Reuman, D. C. The relationship between body mass and field metabolic rate among individual birds and mammals. J. Anim. Ecol. 82(5), 1009-1020 (2013).

[11] Weibel, E. R., Bacigalupe, L. D., Schmitt, B., and Hoppeler, H. Allometric scaling of maximal metabolic rate in mammals: muscle aerobic capacity as determinant factor. Respir. Physiol. Neurobiol. 140(2), 115-132 (2004).

[12] McGill, B. J. and Mittelbach, G. G. An allometric vision and motion model to predict prey encounter rates. Evol. Ecol. Res. 8(4), 691-701 (2006).

[13] Pawar, S., Dell, A. I., and Savage, V. M. Dimensionality of consumer search space drives trophic interaction strengths. Nature 486(7404), 485-489 (2012).

[14] Pawar, S., Dell, A. I., and Savage, V. M. Pawar et al. reply. Nature 493(7434), E2-E3 (2013). 
[15] Pawar, S., Dell, A. I., and Savage, V. M. From metabolic constraints on individuals to the dynamics of ecosystems. In Aquat. Funct. Biodivers. An Ecol. Evol. Perspect., Belgrano, A., Woodward, G., and Jacob, U., editors, 3-36. Elsevier (2015).

[16] Carbone, C., Teacher, A., and Rowcliffe, J. M. The costs of carnivory. PLoS Biol. 5(2), e22, feb (2007).

[17] Carbone, C., Codron, D., Scofield, C., Clauss, M., and Bielby, J. Geometric factors influencing the diet of vertebrate predators in marine and terrestrial environments. Ecol. Lett. 17(12), 1553-9, dec (2014).

[18] Taylor, C. R., Heglund, N. C., and Maloiy, G. M. Energetics and mechanics of terrestrial locomotion. I. Metabolic energy consumption as a function of speed and body size in birds and mammals. J. Exp. Biol. 97(1970), 1-21 (1982).

[19] Carbone, C., Mace, G., Roberts, S., and Macdonald, D. Energetic constraints on the diet of terrestrial carnivores. Nature 402(November), 1997-2000 (1999).

[20] DeLong, J. P. and Vasseur, D. a. A dynamic explanation of size-density scaling in carnivores. Ecology 93(3), 470-476 (2012).

[21] Wilson, R. P., Griffiths, I. W., Legg, P. A., Friswell, M. I., Bidder, O. R., Halsey, L. G., Lambertucci, S. A., and Shepard, E. L. C. Turn costs change the value of animal search paths. Ecol. Lett. 16(9), 1145-1150 (2013).

[22] Blake, R. W. and Domenici, P. Biomechanics in animal behaviour. BIOS Scientific, Oxford, (2000).

[23] Kiltie, R. A. Scaling of visual acuity with body size. Funct. Ecol. 14(2), 226-234 (2000).

[24] Field, D. J. Relations between the statistics of natural images and the response properties of cortical cells. J. Opt. Soc. Am. A. 4(12), 2379-2394 (1987).

[25] Vucic-pestic, O., Rall, C., Kalinkat, G., Brose, U., Rall, B. C., Kalinkat, G., and Brose, U. Allometric functional response model: Body masses constrain interaction strengths. $J$. Anim. Ecol. 79(1), 249-256 (2010).

[26] De Vries, J. L., Pirk, C. W. W., Bateman, P. W., Cameron, E. Z., and Dalerum, F. Extension of the diet of an extreme foraging specialist, the aardwolf (Proteles cristata). African Zool. 46(1), 194-196 (2011).

[27] Van Valkenburgh, B. Deja vu: the evolution of feeding morphologies in the Carnivora. Integr. Comp. Biol. 47(1), 147-163, jul (2007).

[28] Dell, A. I., Pawar, S., and Savage, V. M. Temperature dependence of trophic interactions are driven by asymmetry of species responses and foraging strategy. J. Anim. Ecol. 83(1), 70-84, may (2014).

[29] Shipley, L. A., Gross, J. E., Spalinger, D. E., Hobbs, N. T., and Wunder, B. A. The scaling of intake rate in mammalian herbivores. Am. Nat. 143(6), 1055-1082 (1994).

[30] Ramesh, T., Kalle, R., Sankar, K., and Qureshi, Q. Role of body size in activity budgets of mammals in the Western Ghats of India. J. Trop. Ecol. 31(JUNE), 315-323 (2015).

[31] Rowcliffe, J. M., Kays, R., Kranstauber, B., Carbone, C., and Jansen, P. A. Quantifying levels of animal activity using camera trap data. Methods Ecol. Evol. 5(11), 1170-1179, sep (2014). 
[32] Savage, V. M. and West, G. B. A quantitative, theoretical framework for understanding mammalian sleep. Proc. Natl. Acad. Sci. U. S. A. 104(3), 1051-1056 (2007).

[33] Holling, C. S. The functional response of invertebrate predators to prey density. Mem. Entomol. Soc. Canada 48(48), 1-86 (1966).

[34] Huwaldt, J. A. Plot Digitizer, (2014).

[35] Gittleman, J. L. Carnivore Behavior, Ecology, and Evolution. Chapman \& Hall Ltd, London, 1st edition, (1989).

[36] Tucker, M. A. and Rogers, T. L. Examining predator-prey body size, trophic level and body mass across marine and terrestrial mammals. Proc. R. Soc. B 281(October), 20142103 (2014).

[37] R Development Core Team. R: A language and Environment for Statistical Computing. $\mathrm{R}$ Foundation for Statistical Computing, Vienna, Austria, (2015).

[38] Felsenstein, J. Phylogenies and the Comparative Method. Am. Nat 125(125), 3-147 (1985).

[39] Nyakatura, K. and Bininda-Emonds, O. R. P. Updating the evolutionary history of Carnivora (Mammalia): a new species-level supertree complete with divergence time estimates. BMC Biol. 10, 12-43 (2012).

[40] Harmon, L., Weir, J., Brock, C., Glor, R., and Challenger, W. Geiger: investigating evolutionary radiations. Bioinformatics 24, 129-131 (2008).

[41] Muggeo, V. M. R. segmented: An R package to Fit Regression Models with Broken-Line Relationships. R News 8(May), 20-25 (2008).

[42] Pinheiro, J., Bates, D., DebRoy, S., Sarkar, D., and R Core Team. nlme: Linear and Nonlinear Mixed Effects Models, (2017). R package version 3.1-131.

[43] von Buddenbrock, W. Über die kinetische und statische Leistung großer und kleiner Tiere und ihre Bedeutung für den Gesamtstoffwechsel Naturwissenschaften 40, 675-680 (1934). 